\title{
FINDING FIBRE FACES IN FINITE COVERS
}

\author{
D. D. LONG AND A. W. REID
}

\section{Introduction}

A well-known conjecture about closed hyperbolic 3-manifolds asserts that the first Betti number can be increased without bound by passage to finite sheeted covers. If the manifold is fibred, it is not difficult to see that a strengthening of this conjecture is that the number of fibred faces (see $\S 2.1$ for the definition of a fibred face) of the unit ball of the Thurston norm can be made arbitrarily large by passage to finite sheeted covers. The main result of this note is the following.

Theorem 1.1. Suppose that $M$ is a closed arithmetic hyperbolic 3-manifold which fibres over the circle.

Then given any $K \in \mathbf{N}$, there is a finite sheeted covering of $M$ for which the unit ball of the Thurston norm has $>K$ fibred faces.

A consequence of Theorem 1.1 (see $\S 2$ for a proof) is:

Corollary 1.2. Let $M$ be a closed arithmetic hyperbolic 3-manifold that fibres over the circle. Then the rank of its second homology can be increased without bound.

While this follows from a stronger result proved in [4] (subsequently reproved in [1] and [11]), namely that the conclusion of Corollary 1.2 holds for an arbitrary closed arithmetic hyperbolic 3-manifold with positive first Betti number, the proof given here is somewhat different.

The proof of Theorem 1.1 is purely geometric, using ideas of [3], and the density of the commensurator of an arithmetic Kleinian group. The first example of this phenomenon was recently given by Dunfield and Ramakrishnan [6], also using arithmetic hyperbolic 3-manifolds, but appealing to quite sophisticated number theoretic aspects of these manifolds.

Explicit small examples of arithmetic hyperbolic 3-manifolds which fibre over the circle are known, see e.g. the first example of [3] as analysed in [9] and described briefly in $\S 3$. Many other examples are provided by the tables of [2].

\section{Proof of the main result}

2.1. We begin with a preliminary discussion of some facts about bundles that will be needed. All manifolds are oriented.

Let $M$ be a closed hyperbolic 3-manifold that fibres of the circle with pseudoAnosov monodromy $\phi$ and fibre $F$. Associated to $\phi$ is the suspension flow on $M$, denoted by $\mathcal{F}_{\phi}$, and is constructed as the image in $M$ of the foliation of the product $F \times I$ by lines.

Received by the editors June 27, 2007. 
Recall that Thurston shows in $[10]$ that the set $\mathcal{C}$ of cohomology classes in $\mathrm{H}^{1}(\mathrm{M} ; \mathbf{R})$ represented by non-singular closed 1 -forms is some union of the cones on open faces of the unit ball of the Thurston norm minus the origin. Furthermore, the set of elements in $\mathrm{H}^{1}(\mathrm{M} ; \mathbf{Z})$ whose Poincare duals are represented by fibres consists of the set of all lattice points in $\mathcal{C}$. Thus if $M$ is fibred with monodromy $\phi$ we can associate the (open) face $T(\phi)$ to $\phi$. We call $T(\phi)$ a fibre face. The following result is proved by Fried (see Theorem 7 of [7]) and provides the connection from flows to fibre faces of the unit ball of the Thurston norm.

Theorem 2.1. Let $M$ be a closed hyperbolic 3-manifold that fibres over $S^{1}$ and for which $\mathrm{H}^{1}(\mathrm{M} ; \mathbf{Z}) \neq \mathbf{Z}$. The face $T(\phi)$ determines $\mathcal{F}_{\phi}$ up to strict conjugacy (that is to say, the flows are conjugate via a diffeomorphism isotopic to the identity map).

2.2. Before giving the proof of Theorem 1.1 we make a simple observation that will be helpful.

Let $X=\mathbf{H}^{3} / \Gamma$ be a closed hyperbolic 3-manifold, and let $S \hookrightarrow X$ be a $\pi_{1}$-injective oriented immersion of a quasi-Fuchsian surface whose lift to the universal covering is an oriented embedding $\widetilde{S} \rightarrow \widetilde{X}$. We consider the components of $\widetilde{S}$ being oriented with a positive side and negative side given by such a designation on $S$.

We claim the following.

Lemma 2.2. Fix disjoint open sets $U$ and $V$ in the 2 -sphere at infinity, whose complement contains an open set.

Then there are disjoint translates $\widetilde{S_{1}}$ and $\widetilde{S_{2}}$ in $\widetilde{S}$ with the properties that

- $\partial \widetilde{S_{1}} \subset U$

- $\partial \widetilde{S_{2}} \subset V$

- The positive sides of $\widetilde{S_{1}}$ and $\widetilde{S_{2}}$ face each other.

Proof. Let $W$ be an open set which lies in the complement of $U \cup V$. We can find a hyperbolic element $\gamma \in \Gamma$ such that its attracting fix point lies in $W$, and thus arrange that there is a component of $\widetilde{S}$, denoted $\widetilde{S}^{\prime}$, with $\partial \widetilde{S}^{\prime} \subset W$. Now standard dynamical properties of hyperbolic elements in $\Gamma$ allow us to find two hyperbolic elements $\gamma_{1}$ and $\gamma_{2}$ with the property that each has one fix point inside the disc spanned by $\partial \widetilde{S}^{\prime} \subset W$ and further so that the other fix point of $\gamma_{1}$ lies in $U$ and the other fix point of $\gamma_{2}$ lies in $V$.

Now by applying sufficiently high powers of the hyperbolic elements $\gamma_{i}$ 's we see that we achieve the situation of the lemma.

\section{Proof of Theorem 1.1.}

We begin by showing how to obtain two fibre faces for the Thurston norm ball, since this illustrates the main idea. By assumption $M=\mathbf{H}^{3} / \Gamma$ fibres over the circle, and we let the associated suspension flow be denoted by $\mathcal{F}_{1}$.

It follows from [8], Theorem 1.1 (see also Lemma 5.8 therein), that one can find an immersion $S \hookrightarrow M$ transverse to the flow $\mathcal{F}_{1}$ (and therefore necessarily $\pi_{1}$-injective) which is quasi-Fuchsian. It follows from [3] (see the discussion on p. 264 following the proof of Lemma 3.3) that the lift of this immersion to the universal covering is embedded. Hence, by Lemma 2.2 , there is a pair of disjoint lifts, $\widetilde{S_{1}}$ and $\widetilde{S_{2}}$ whose positive sides face each other. Thus the negative sides of these lifts define a pair of disjoint open discs in the 2-sphere at infinity, which we will refer to informally as caps. 
Choose any closed flowline of $\mathcal{F}_{1}$ and let $\gamma$ be a lift of this closed flowline to $\mathbf{H}^{3}$. By standard arguments, there is an element $g \in \operatorname{PSL}(2, \mathbf{C})$ which maps one of the endpoints of $\gamma$ into one cap, and the other endpoint of $\gamma$ into the other. Thus $g(\gamma)$ projects to a closed flowline of a flow on $\mathbf{H}^{3} / g \Gamma g^{-1}$, which has one of its endpoints in one cap and one in the other.

Since $M$ is arithmetic, the commensurator of $\Gamma$ is dense in $\operatorname{PSL}(2, \mathbf{C})$. Hence, by adjusting $g$ slightly, we can assume in addition that $g \Gamma g^{-1}$ is commensurable with $\Gamma$. Let $\Gamma_{12}$ be the intersection of these two subgroups, $M_{12}$ the cover of $M$ determined by $\Gamma_{12}$ and let $\mathcal{F}_{2}$ be the conjugated flow on $\mathbf{H}^{3} / g \Gamma g^{-1}$ lifted to $M_{12}$. Lift the original flow $\mathcal{F}_{1}$ to $M_{12}$, where to avoid clumsy notation, we continue to denote it by $\mathcal{F}_{1}$.

We claim that one cannot isotope $\mathcal{F}_{2}$ to $\mathcal{F}_{1}$ in $M_{12}$. The reason is this. By construction, $g(\gamma)$ covers some closed flowline of $\mathcal{F}_{2}$. However the positioning of the endpoints of $g(\gamma)$ ensures that any loop isotopic to the closed flowline must meet the two chosen lifts of the $\mathcal{F}_{1}$-transverse surface $S$ in opposite orientations. This implies that the loop cannot be isotoped into the flow $\mathcal{F}_{1}$. By Theorem 2.1, these flows represent different faces of the unit ball of the Thurston norm as required.

The general case is similar: We now work on $M_{12}$. Fix some quasi-Fuchsian immersion $G$ transverse to the flow $\mathcal{F}_{2}$. Taking $U$ and $V$ to be the cap regions defined by $\widetilde{S_{1}}$ and $\widetilde{S_{2}}$, we apply Lemma 2.2 to find disjoint lifts of $G, \widetilde{G_{1}}$ and $\widetilde{G_{2}}$ with boundaries inside the $S$-caps and with positive sides facing each other. This gives new, smaller, $G$-caps into which we may place the endpoints of a flowline using an element of the commensurator. An identical argument now shows that the new flow this defines cannot be strictly conjugate to either of the first two flows.

Proof of Corollary 1.2. By Theorem 1.1, we may find a finite sheeted covering $p: \widetilde{M} \longrightarrow M$ for which the number of fibred faces in the Thurston norm of $\widetilde{M}$ is strictly larger than the number of fibred faces in $M$. Suppose that this covering does not increase the rational dimension of $\mathrm{H}_{2}$, so that $p_{*}$ is a rational isomorphism.

The preimages of fibres are fibres and are therefore norm-minimising, so that since the dimension of $\mathrm{H}_{2}$ is not increased, the interior of any top-dimensional face of the Thurston norm ball of $M$ which corresponds to fibrations lifts into a top-dimensional face of the Thurston norm ball of $\widetilde{M}$ corresponding to fibrations. In particular, such faces cannot be subdivided.

Moreover, if a class in $H_{2}(M)$ is represented by an embedded incompressible connected surface $S$ which is not the fibre of a fibration of $M$, then its preimage $p^{-1}(S)$ cannot be homologous to the fibre of a fibration of $\widetilde{M}$. The reason is this: If $F$ is a fibre of $\widetilde{M}$, with $[F]=\left[p^{-1}(S)\right] \in H_{2}(\widetilde{M})$, then using the dual cohomology classes $[F]=\left[p^{-1}(S)\right] \in H^{1}(\widetilde{M})$ to form an infinite cyclic covering, we see the incompressible surface $p^{-1}(S)$ lifts to the product $F \times \mathbf{R}$, since the cohomology class is given by algebraic intersection number of classes in $H_{1}(\widetilde{M})$ with either of $F$ or $p^{-1}(S)$. Standard 3-manifold topology now implies that $p^{-1}(S)$ and $F$ are isotopic, a contradiction.

Taking these two facts together, we see that fibre faces lift to fibre faces.

We may now conclude the proof of the corollary: Since there are more fibred faces in $\widetilde{M}$ than in $M$, this forces there to be a top-dimensional face in $M$ which contains a primitive class whose embedded connected incompressible representative $S$ is not a fibre of $M$, but whose preimage $p^{-1}(S)$ has $\left[p^{-1}(S)\right] \in H_{2}(\widetilde{M})$ lies in a fibre face, a contradiction. 


\section{Example 1 of $[3]$}

Example 1 of [3] is described explicitly using a branched flat structure, however from the discussion in [3] this example can easily be seen to be described as follows.

Let $T$ be the 1-punctured torus bundle over the circle with monodromy $R^{2} L^{2}$. Fix a framing for the boundary torus so that a longitude is the boundary of a fiber, and a meridian taken as the suspension of a point on the boundary of a fiber. Then $(0,2)$ orbifold filling on $T$ provides a 2-orbifold bundle $Q$ over the circle. The genus 2 bundle $M$ described in [3] is a finite cover of this. As shown in [3] this genus 2 bundle admits a cut and cross join surface that is quasi-Fuchsian. Thus we need to check that this example is arithmetic.

This can be verified directly by using Snap [5] to check that $Q$ is arithmetic. The invariant trace-field is $\mathbf{Q}(\sqrt{-3})$ and the invariant algebra is ramified at the places above 2 and 3 .

Remark: In addition the bundle $Q$ is also easily seen to be commensurable with the 2 -orbifold bundle described in $\S 4.2$ of [9].

\section{Acknowledgements}

The authors thank Nathan Dunfield reading the original manuscript and for offering some useful comments which clarified the exposition.

Both authors supported in part by grants from the NSF.

\section{References}

[1] I. Agol, Virtual Betti numbers of symmetric spaces, Preprint.

[2] J. O. Button, Fibred and virtually fibred hyperbolic 3-manifolds in the censuses, Experimental Math. 14 (2005), 231-255.

[3] D. Cooper, D. D. Long and A. W. Reid, Bundles and finite foliations, Invent. Math. 118 (1994), 255-283.

[4] - On the virtual Betti numbers of arithmetic hyperbolic 3-manifolds, Preprint

[5] D. Coulson, O. A. Goodman, C. D. Hodgson and W. D. Neumann, Computing arithmetic invariants of 3-manifolds, Experimental Math. 9 (2000), 127-152.

[6] N. Dunfield and D. Ramakrishnan, Increasing the number of fibered faces of arithmetic hyperbolic 3-manifolds, Preprint in preparation.

[7] D. Fried, Fibrations over $S^{1}$ with pseudo-Anosov monodromy, Exposé 14, Asterisque 66-67 (1979).

[8] J. D. Masters, Thick surfaces in hyperbolic 3-manifolds, Geom. Dedicata 119 (2006), 17-33.

[9] A. W. Reid, A non-Haken hyperbolic 3-manifold covered by a surface bundle, Pacific J. Math. 167 (1995), 163-182.

[10] W. P. Thurston, A norm for the homology of 3-manifolds, Mem. Amer. Math. Soc. 339 (1986), 99-130.

[11] T. N. Venkataramana, Virtual Betti numbers of compact locally symmetric spaces, Preprint.

Department of Mathematics, University of California, Santa Barbara, CA 93106

E-mail address: long@math.ucsb.edu

Department of Mathematics, University of Texas, Austin, TX 78712

E-mail address: areid@math.utexas.edu 